\title{
Analgesia in the accident and emergency department: do SHOs have the knowledge to provide optimal analgesia?
}

\author{
S Sandhu, P Driscoll, J Nancarrow, D McHugh
}

\begin{abstract}
Objective-To assess senior house officers' knowledge in prescribing emergency analgesia for acute presentations in the accident and emergency (A\&E) department.

Design-Prospective telephone survey of a defined population of SHOs, using a standardised structured questionnaire, in the months of October and November, 1995; 231 SHOs from 215 A\&E departments were interviewed. The questionnaire required responses to hypothetical scenarios. A six member expert panel from the local region was consulted for suggestions for appropriate responses.
\end{abstract}

Main outcome measures-Comparisons between SHO responses and those of an expert panel.

Results-For choice of analgesic agent, $83 \%$ of SHO responses were appropriate, for route of administration $57 \%$, and for the dose of drug $34 \%$. The scenario with the best overall response was a sprained ankle. The paediatric case with partial burns faired worse. Responses to a myocardial infarction scenario were the most consistent.

Conclusions-A\&E SHOs lack knowledge and confidence when asked to prescribe emergency analgesia for acute conditions. Responses to certain scenarios were extremely varied, indicating a need for national analgesia guidelines and protocols. Recognised training in pain management should be more readily available. (F Accid Emerg Med 1998;15:147-150)

Department of

Emergency Medicine,

Hope Hospital, Eccles

Old Road, Salford,

Manchester M6 8HD,

UK

S Sandhu

P Driscoll

J Nancarrow

D McHugh

Correspondence to:

Mr Peter Driscoll.

Accepted

for publication

24 September 1997 pain as part of their complaint. ${ }^{1}$ management should therefore be a common activity because it helps patients develop a more normal physiological status as well as facilitating the clinical assessment and the doctor-patient relationship. ${ }^{3}$

A United States study found that $56 \%$ of patients with acutely painful medical and surgical conditions received no analgesia while in the A\&E department. ${ }^{4}$ Furthermore, $32 \%$ of the patients receiving analgesia had a less than the optimal dose. However, it could be argued that these conclusions are not valid because the study population suffered from many different conditions and some were paediatric cases. Nevertheless corroborative evidence comes from a later American study by Selbst and Clark. ${ }^{5}$ They found that $60 \%$ of A\&E patients reviewed received no analgesia for their acutely painful conditions, $12 \%$ received a suboptimal initial dose, and children were significantly less likely to receive analgesia than adults.

In 1986, Reichl and Bodiwala ${ }^{6}$ used seven common hypothetical situations to evaluate pain management by United Kingdom $A \& E$ senior house officers (SHOs). They concluded that analgesia management by these doctors was inadequate. They also suggested the need for more detailed teaching on pain management, analgesia oriented induction courses, and the adoption of an appropriate analgesia policy in A\&E departments. Since 1986 there have been major developments in A\&E departments in the United Kingdom. Induction courses and active education programmes for SHOs are now standard. Thus the previously defined lack of knowledge may have been corrected. This study was designed to assess the current analgesic knowledge of A\&E SHOs.

\section{Methods}

A prospective telephone survey of $A \& E$ departments on the mainland was conducted in October and November, 1995. In each case an explanation of the study was given, along with an invitation to any one SHO present in the department at the time to complete the questionnaire. If departments were not particularly busy, and if invited to do so by the participating SHO, other available A\&E SHOs were interviewed as well. We decided that departments should be contacted a maximum of five times. If the department was too busy, an appropriate time for subsequent communication was ascertained on first contact.

\section{STUDY POPULATION}

We identified 559 A\&E departments using the British Association for Accident and Emergency Medicine (BAAEM) directory, $1993 .^{7}$ 
Table 1 Choice of analgesia for all scenarios

\begin{tabular}{|c|c|c|c|c|c|c|c|}
\hline \multirow[b]{2}{*}{ Drug group } & \multicolumn{7}{|c|}{ Scenario } \\
\hline & $1 a$ & $1 b$ & 2 & 3 & 4 & 5 & 6 \\
\hline Strong opioid & 210 & 158 & 192 & 1 & 179 & 231 & 168 \\
\hline Codeine compound & & 1 & 1 & 64 & & & \\
\hline Paracetamol/aspirin & & & & 27 & 1 & & 19 \\
\hline NSAIDs & 19 & 43 & 36 & 122 & 8 & & 4 \\
\hline Non-opioids and/or NSAIDs & & & & 13 & & & 1 \\
\hline Nitrous oxide & & 7 & & & 1 & & \\
\hline Sedatives & & & & 1 & & & 1 \\
\hline Other oral & & & & & 2 & & \\
\hline Nerve blocks & 2 & 2 & 1 & & & & \\
\hline Don't know & & 1 & & & & & 9 \\
\hline None & & 19 & 1 & 3 & 40 & & 29 \\
\hline Adjuvant treatment & 13 & 18 & 9 & & 1 & & 5 \\
\hline
\end{tabular}

See appendix for description of scenarios; NSAID, non-steroidal anti-inflammatory drug Classification of responses

Strong opioids: morphine, diamorphine, pethidine.

Codeine compounds: co-codamol, co-dydramol, co-proxamol.

Non-steroidal anti-inflammatory drugs (NSAIDs): ibuprofen, diclofenac sodium, naproxen

Non-opioids and/or NSAlDs: paracetamol and/or ibuprofen, co-codamol and naproxen, co-dydramol/ibuprofen.

Sedatives: diazepam, vallergan.

Other oral: antispasmodic.

Adjuvant treatment: local anaesthetic, sedative, entonox.

Only departments with SHOs were contacted. Ministry of Defence, children's, and eye hospitals were excluded because SHOs working there may not have regular exposure to the conditions described in the questionnaire. Thus we did not consider these departments representative of those found in the rest of the United Kingdom.

\section{QUESTIONNAIRE}

We constructed a standardised structured questionnaire, based on a similar study by Reichl and Bodiwala. ${ }^{6}$ Six hypothetical clinical scenarios were devised to determine which analgesic drug, dose, and route of administration the SHO would choose. These scenarios are described in the appendix. To provide a fair and balanced representation of $A \& E$ attenders, the questions took account of the variation of age, sex, and acutely painful conditions presenting to $A \& E .^{3}$

To obtain information on previous training in analgesia prescribing, the questionnaire also asked about previous experience in $A \& E$ medicine (at least six months), anaesthesia, and the completion of the advanced trauma life support (ATLS) course.

\section{DATA COLLECTION}

We recorded all the SHOs' responses. If they replied with generalised groups-for example opioids-they were asked if they would favour a particular drug. If more than one drug was contemplated, a personal preference was

Table 2 Choice of route of analgesic administration

\begin{tabular}{|c|c|c|c|c|c|c|c|}
\hline \multirow[b]{2}{*}{ Route } & \multicolumn{7}{|c|}{ Scenario } \\
\hline & $1 a$ & $1 b$ & 2 & 3 & 4 & 5 & 6 \\
\hline Intravenous & 123 & 111 & 77 & 1 & 82 & 231 & 73 \\
\hline Intramuscular & 104 & 83 & 148 & 10 & 102 & & 51 \\
\hline Rectal & 2 & 4 & 1 & 2 & 2 & & 1 \\
\hline Oral & & 1 & 1 & 212 & 1 & & 58 \\
\hline Inhaled & & 7 & & & & & \\
\hline Nerve block & 2 & 1 & 1 & & & & \\
\hline Subcutaneous & & 1 & & & 1 & & \\
\hline Don't know & & 4 & 2 & 3 & 2 & & 19 \\
\hline None & & 19 & 1 & 3 & 40 & & 29 \\
\hline
\end{tabular}

See appendix for description of scenarios sought. If a comparable situation arose regarding dose of drug, or if a dose was not given, the SHOs were asked what they would do. Similar inquiries were made about the route of administration. Where an acceptable range of drug dose was given, it was assumed that the respondent would titrate the dose according to effect. Intravenous access was assumed to be possible in all patients if needed.

\section{EXPERT PANEL}

An expert panel was consulted to determine appropriate answers for choice of drug, route of administration, and dose to achieve sufficient analgesia for the painful conditions in each of the scenarios. The six membered panel included two consultants in A\&E medicine, a consultant in anaesthesia, a consultant in paediatric emergency medicine, and two specialist registrars in A\&E medicine (both of whom had had formal training in anaesthesia).

\section{ANALYSIS}

The responses were recorded on a questionnaire by hand and then stored and analysed on an IBM compatible statistical software package (SPSS for Windows) at the Computer Microlab, Manchester Medical School. The responses of the SHOs were then compared with those of the expert panel and classed as "appropriate," "inappropriate," "don't know," or "no analgesia prescribed."

Appropriate statistical methods were applied where necessary. It was expected that these would mainly be cross tabulations. Analysis was considered statistically significant if probability (p) was less than 0.05 .

\section{Results}

STUDY POPULATION

All the 217 A\&E departments identified for the study were contacted. In two cases SHOs were contacted a total of five times, but their departments were repeatedly busy and as a result were unable to take part in the study. Locums were included if they had worked regularly in the department for at least one month $(n=3)$. Sixteen additional SHOs took part as their departments were not particularly busy at the time of the interview. This gave an overall study population of 231 .

Overall, 1515 responses recommended the administration of analgesia, of a possible total of 1617 responses $(93.7 \%)$. Most of these were strong opioids $(70.4 \%)$. The main routes of administration were intravenous $(43.2 \%)$ and intramuscular $(30.8 \%)$. A breakdown of analgesics and the routes by which they would be given are shown in tables 1 and 2 .

\section{OVERALL APPROPRIATENESS OF RESPONSES}

Comparing the SHOs' responses with those of the expert panel (table 3), the choice of drug was considered appropriate in 1337 cases $(82.7 \%)$. Route of administration was appropriate in 919 of cases $(56.8 \%)$, and the dose of drug in $544(33.6 \%)$. The myocardial infarction scenario had the best responses for both the prescribing of an appropriate analgesic drug and the choice of a suitable route. 
Table 3 Expert panel's opinion

\begin{tabular}{llll}
\hline Scenario & Drug & Route & Dose \\
\hline 1a & Strong opioid & IV & Titrated to effect \\
1b & Strong opioid & IV & Titrated to effect \\
2 & Strong opioid & IV & Titrated to effect \\
3 & Codeine compound and/or NSAID & PO/IM & As standard dose \\
4 & Strong opioid & IV & Titrated to effect \\
5 & Strong opioid & IV & Titrated to effect \\
6 & Strong opioid & IV & Titrated to effect \\
\hline
\end{tabular}

IM, intramuscular; IV, intravenous; NSAID, non-steroidal anti-inflammatory drug; PO, orally.

Table $4 p$ Values for $\chi^{2}$ tests

\begin{tabular}{llll}
\hline & $\begin{array}{l}\text { Previous AEE } \\
\text { experience }\end{array}$ & $\begin{array}{l}\text { Previous anaesthetic } \\
\text { experience }\end{array}$ & ATLS certificate \\
\hline Analgesic drug & 0.131 & 0.812 & 0.085 \\
Route of administration & 0.009 & 0.571 & 0.051 \\
Dose of analgesic drug & 0.095 & 0.749 & 0.009 \\
\hline
\end{tabular}

ATLS, advanced trauma life support course

However, the responses for dose of drug in this scenario were only appropriate in 125 of 231 cases $(54.1 \%)$. The scenario with the best overall response was that of the sprained ankle. Here the correct drug was prescribed 199 times $(86.1 \%)$. The appropriate route was used by $222(96.1 \%)$, and the correct dose supplied by $198(85.7 \%)$. The paediatric case with partial burns faired worse, with 168 $(72.7 \%)$ prescribing a suitable drug, 73 (31.6\%) giving it correctly, and only $12(5.2 \%)$ giving an acceptable dose.

The case with the least appropriate responses for analgesic drug $(158,68.4 \%)$ was the compound fracture of the tibia and fibula (1b in the appendix). Of those who initially prescribed appropriately, $139(66.2 \%)$ continued with a suitable choice 20 minutes later. Of the respondents whose initial prescription was inappropriate, $19(90.5 \%)$ prescribed more appropriately for treating continuing pain after 20 minutes. Only two respondents (9.5\%) continued to prescribe inadequately. Eighteen $(90 \%)$ of those who gave no analgesia 20 minutes later had already prescribed appropriately.

\section{PREVIOUS EXPERIENCE}

The study population consisted of 76 SHOs with previous experience in A\&E (32.9\%), 13 with previous experience in anaesthesia $(5.6 \%)$, and 41 stating that they had completed the ATLS course $(17.7 \%)$. These three groups were not mutually exclusive.

The subpopulations were individually compared with the rest of the population using $\chi^{2}$ tests, to see if there were any significant differences in response (table 4). In the choice of route of administration, the group with previous experience in $\mathrm{A} \& \mathrm{E}$ were significantly better. ATLS trained SHOs were best at choosing the dose of analgesic drug.

\section{Discussion}

Our aim in this study was to explore the use of analgesia by junior doctors in the A\&E department. We showed that while the great majority of respondents chose an appropriate analgesic drug, they often did not choose an appropriate route. More significantly, the dose was often wholly inadequate.
The types of response indicate that many SHOs lack confidence in using strong opioids in adults and children. In addition few doctors would titrate the analgesic against the patient's response, and the majority were reluctant to use the intravenous route in the elderly. The greatest variety of drugs prescribed was for the sprained ankle (27 in all), suggesting personal choices led to an array of prescriptions. However, from interviewing the SHOs it transpired that they tended to use "whatever is available in the department at the time." Consequently prescribing was not entirely due to personal choice, an area which has been identified by Yates et al. ${ }^{8}$

We also showed that the group with previous $\mathrm{A} \& \mathrm{E}$ experience was better at choosing the route of administration, and the group with the ATLS certificate was better at choosing the dose of analgesic drug. It is not clear what part of the ATLS training made this group better in some aspects of their response. Because of the small numbers in the group with experience in anaesthesia, the results may not reflect true statistical differences. Further research is needed to clarify this issue.

Driscoll et al suggest that the reason for prescribing no analgesia is poor objective estimation of the degree of pain suffered by the patient. ${ }^{3}$ "Psychic numbing" can develop through repetitive exposure to complaints of pain. A review article on acute pain relief by Sutcliffe ${ }^{9}$ suggests further possible reasons for inadequate pain relief. These include a lack of understanding of pain, inadequate knowledge of the pharmacology of drugs and adjuvant techniques, little skill in administration, failure of pain assessment and communication, and fears about addiction, overdose, side effects, and the masking of physical signs.

\section{DESIGN OF THE STUDY}

The only way to assess current knowledge is by a cross sectional (prevalence) survey. The design of our study allowed the knowledge of SHOs to be assessed and compared with the previous work done by Reichl and Bodiwala. ${ }^{6}$ A telephone survey was chosen because it incorporates the advantages of both written questionnaires and personal interviews. Telephone surveys are cost-effective and accurate and they assure a high response rate. ${ }^{10}$ The possibility of variation between subjects was reduced by providing a full explanation of the study, an estimation of how long it was going to take (around five minutes), and an option to phone back at a more convenient time (if possible). Any observer variation was reduced by the use of a structured questionnaire.

A limitation of the study is the selection of the sample from the parent population. While a simple random sample of SHOs would have been ideal, the difficulty of selecting such a group was that a complete telephone directory of currently registered A\&E SHOs would have been hard to obtain as they continually rotate every six months. The method chosen ensured a high response rate and good quality information. We believe the sample was random, as there was no self selection and SHOs who were 
hard to identify for the complete (national) telephone questionnaire were not omitted.

\section{RECOMMENDATIONS}

This study shows there is a clear need for a draft document setting out appropriately constituted guidelines on pain management in the A\&E department. These need to cover the "chain of analgesia," that is: the optimum drug, by the optimum route, by the optimum dose, to achieve the optimum effect. This correct order is essential for effective analgesia. While the results of the study are encouraging in that most SHOs seem to give an appropriate drug, they are less so with respect to the route of administration and dose. Only by considering all these aspect can we effect policies for optimal pain management.

Recently published articles outline recommendations on the development of protocols for pain management. ${ }^{11-15}$ These include the following: extensive review of scientific reports, establishment of practice patterns, identification of problem areas, promotion of research, review of current protocols and development of new ones with respect to specific criteria (for example, sensitivity, specificity, reliability, reproducibility, clinical applicability, flexibility, and clarity), a multidisciplinary approach, implementation of guidelines with appropriate validation, extensive research with the publication of both positive and negative outcomes, and finally a review of policies so that they can be altered or abolished as necessary.

There is therefore much work to be done to ensure that accident and emergency patients receive optimum pain relief.

We acknowledge the assistance of S Morris, MRPharmS.

\section{Appendix}

\section{Questionnaire for A\&E SHOs}

What analgesia would you use in the following scenarios?

(la) A 23 year old female with a compound fracture of the tibia and fibula in severe pain

- normal procedures practised

- $60 \mathrm{~kg}$, no medical problems. (1b) The above patient still in moderate to severe pain 20 minutes later.

(2) An 80 year old female with a fractured neck of femur with pain at rest:

- frail, pain at rest.

- $55 \mathrm{~kg}$, no major medical problems.

(3) An 18 year old male with a sprained ankle in pain: - sports injury, difficulty in bearing weight

- no underlying fractures

- insisting analgesia

- $70 \mathrm{~kg}$, no major medical problems.

(4) A 45 year old male with severe abdominal pain:

- not colicky, abdomen rigid

- haemodynamically stable

- surgeons busy for 30 minutes

- $80 \mathrm{~kg}$, no medical problems.

(5) A 60 year old male with an acute myocardial infarction in pain:

- haemodynamically stable

- $75 \mathrm{~kg}$, no major medical problems

(6) A 2 year old female with $15 \%$ partial thickness burns in distress:

- no burns to face

- $12 \mathrm{~kg}$, no medical problems

- Intravenous access attainable.

1 Directory of emergency and special care units 1995. Cambridge: CMA Medical Data, 1995.

2 Boisaubin E. The assessment and treatment of pain in the emergency room. Clin J Pain 1989;5(suppl 2):S19-25.

3 Driscoll P, Gwinnutt C, Nancarrow, J. Analgesia in the emergency department. Pain Rev 1995;2:185-200.

4 Wilson J, Pendleton J. Oligoanalgesia in the emergency department. Am J Emerg Med 1989;7:620-3.

5 Selbst S, Clark M. Analgesic use in the emergency department. Ann Emerg Med 1990;19:1010-13.

6 Reichl M, Bodiwala G. Use of analgesia in severe pain in the emergency department. Arch Emerg Med 1987;4:25-31.

7 Royal College of Surgeons of England. British Association for Accident and Emergency Medicine directory 1993. London: Royal College of Surgeons, 1993.

8 Yates D, Laing G, Peters K, Kumar K. Mild analgesics and the accident and emergency department - cost and safety more important than potency? Arch Emerg Med 1984;1: 197-203.

9 Sutcliffe A. Pain relief for acutely ill and injured patients. Care of the Critically Ill 1993;9:266-8.

10 Kidder L, Judd C. Questionnaires and interviews: overview of strategies. In: Research methods in social relations. New York: CBS Publishing Japan, 1986:219-35.

11 Ducharme J. Proceedings of the First International Symposium on Pain Research in Emergency Medicine. Foreword. Ann Emerg Med 1996;27:399-403.

12 Afilalo M, Cantees K, Ducharme J. Current pain-control practices and research. Ann Emerg Med 1996;27:404-7.

13 Terndrup T. Establishing pain policies in emergency medicine. Ann Emerg Med 1996;27:408-11.

14 Cordell W. Pain-control research opportunities and future directions. Ann Emerg Med 1996;27:474-8.

15 Goodacre S, Roden R. A protocol to improve analgesia use in the accident and emergency department. J Accid Emerg Med 1996;13:177-9.

\title{
Managing meningococcal disease: reducing mortality in children and adults
}

\author{
Monday 15 June 1998, Royal College of Physicians, London
}

This national conference, organised by the Meningitis Research Foundation, aims to bring together both paediatric and adult hospital doctors who are involved at all stages in the recognition and treatment of meningococcal disease and to provide a practical learning experience involving case studies, comparison of methods, problems and constraints in treating children, adolescents and adults.

For further information contact:

Meningitis Research Foundation

13 High Street, Thornbury, Bristol BS35 2AE

Tel: $+44(0) 1454281811$

Fax: $+44(0) 1454281094$ 